\title{
GASTO MUNICIPAL EN EDUCACIÓN Y SU EFECTO EN LA DISTRIBUCIÓN DE INGRESOS A NIVEL LOCAL EN CHILE
}

\section{Local Government Expenditure on Education and its Effect on Income Inequality at the County-Level in Chile}

Felipe Manuel Gutiérrez-Garrido Andrés Alberto Acuña-Duarte 


\section{GASTO MUNICIPAL EN EDUCACIÓN Y SU EFECTO EN LA DISTRIBUCIÓN DE INGRESOS A NIVEL LOCAL EN CHILE*}

\section{Local government expenditure on education and its effect on income inequality at the county-level in Chile}

Felipe Manuel Gutiérrez-Garrido y Andrés Alberto Acuña-Duarte ${ }^{b}$

Palabras clave: Desigualdad de ingresos, Gasto público en educación, Respuesta fraccional, Tobit, América Latina.

Keywords: Income inequality, Education expenditure, Fractional response, Tobit, Latin America.

JEL Classification: D63, 124, 015

Received: 30/11/2018

Accepted: 09/12/2019

Published: 28/02/2020 a. Investigador del Departamento de Economía y Finanzas,

Universidad del Bío-Bío, Concepción Chile. fmgutier@egresados.ubiobio.cl Orcid: 0000-0002-0019-3274

b. Investigador del Departamento de Economía y Finanzas,

Universidad del Bío-Bío, Concepción Chile. aacunad@ubiobio.cl

Orcid: 0000-0002-1623-4616

\section{Resumen}

Cuatro medidas de gasto municipal en educación per cápita son utilizadas para analizar su efecto sobre la desigualdad de ingresos a nivel comunal en Chile. La desigualdad es cuantificada mediante los indicadores Gini, Theil, 10/10 y 20/20. El estudio longitudinal consideró 316 comunas para estimar modelos probit de respuesta fraccional para el coeficiente de Gini y Tobit con efectos-aleatorios para los restantes indicadores de desigualdad. La evidencia indica que aumentos en el gasto en educación per cápita reducirían la desigualdad de ingresos, medida con el índice de Theil, pero acentuarían la brecha entre el decil/quintil más rico y el más pobre de la población. Incluso, el mayor gasto en personal educativo incrementaría el coeficiente de Gini y el indicador 10/10. Adicionalmente, el predominio de población indígena y rural exacerbaría la desigualdad comunal. Finalmente, se revela la existencia de una curva de Kuznets convexa para los valores extremos de la distribución de ingreso.

\section{Abstract}

Four measurements of per-capita education expenditure are used to analyze its effect on income inequality at the county-level in Chile. The latter is quantified

* La presente investigación fue desarrollada al interior del grupo de investigación Análisis Económico Sectorial Aplicado, Proyecto GI 160317/EF financiado por la Dirección de Investigación de la Universidad del Bío-Bío, Chile. Los autores agradecen al Ministerio de Desarrollo Social de Chile por haberles permitido disponer de la base de datos de la Encuesta CASEN, y a un evaluador anónimo por sus valiosos comentarios y sugerencias. Todos los resultados e interpretación de los mismos son responsabilidad de los autores. 
through the Gini coefficient, Theil index, and the S80/S2o and S9o/S1o ratios. The longitudinal study considers 316 Chilean counties in order to estimate a fractional response probit model for the Gini coefficient and random-effects Tobit models for the remaining indicators of inequality. Main findings show that an increasing per-capita education expenditure would reduce the income inequality measured by Theil index, but it would accentuate the gap between the poorest and wealthiest decile/quantile. And a greater education expenditure on personnel and teaching staff would enlarge the Gini coefficient and S9o/S1o ratio. In addition, income inequality at the countylevel is exacerbated by a larger indigenous and rural population. Finally, the evidence reveals that a convex Kuznets-curve exists for the extreme values of income distribution.

\section{Introducción}

El crecimiento económico de Chile registró niveles históricos tras la segunda mitad de la década de 1980, siendo una de las economías de mayor crecimiento en América Latina a partir de ese periodo. ${ }^{1}$ Este buen rendimiento macroeconómico es el que ha permitido alcanzar reducciones significativas en los niveles de pobreza en el ámbito nacional, especialmente tras el inicio en 2003 de un ciclo favorable en el precio del cobre (Álvarez, García-Marín, Ilabaca, 2018). ${ }^{2}$ No obstante, los auspiciosos indicadores macroeconómicos no se han traducido en un crecimiento inclusivo, lo que ha generado una alta desigualdad social y una inequitativa distribución de la riqueza, siendo esta última uno de los problemas más importantes de la sociedad chilena tras el retorno a la democracia (Altimir, 1994: Larrañaga, 1999; López y Miller, 2008; Solimano y Torche, 2008). Incluso, la Organización para la Cooperación y el Desarrollo Económico (OCDE, 2015) señala que Chile es en la actualidad el país que exhibe la mayor desigualdad de ingreso al interior del organismo, cuando esta es medida por el coeficiente de Gini.

Por otra parte, existe consenso en que altos niveles de desigualdad impactan negativamente no solo la productividad y el crecimiento económico sostenible, sino también la estabilidad política de un país (Alesina y Perotti, 1996; López y Miller, 2008; Abdullah, Doucouliagos, Manning, 2015). Una fórmula destacada para hacer frente a la inequidad es la mayor inversión en capital humano, que se traduciría en una disminución de la desigualdad de los ingresos, especialmente en las economías en desarrollo (Tinbergen, 1957; Marin y Psacharopoulos, 1976; Winegarden, 1979; Ram, 1989; Ocampo, 1998; Beyer, 1999; De Gregorio y Lee, 2002; Cotte y Cotrino, 2006).

Al respecto, gran parte de la literatura se ha centrado en estimar el retorno de la educación, mientras que solo una fracción menor ha abordado el efecto del gasto público en educación sobre la desigualdad de ingresos. Cabe destacar que la evidencia reportada a nivel agregado (Sylwester, 2002; Keller, 2010; Abdullah et al., 2015) no es concluyente y es contradictoria respecto a la relación negativa entre ambas variables, especialmente en economías emergentes como las latinoamericanas. Adicionalmente, la literatura destaca el rol de los gobiernos locales sobre los niveles de desarrollo del territorio, en particular, sobre la reducción de la pobreza y las desigualdades bajo un contexto de descentralización del poder y una sana gobernanza (Beall Crankshaw, Parnell, 2000; Crook, 2003;

1 Según cifras del Banco Central de Chile, el país exhibió expansiones del producto interno bruto (PIB) incluso por sobre el $10 \%$ durante el periodo 1986-1999, promediando una tasa de crecimiento anual de 6,4\%. Parte de este sobresaliente desempeño económico se ha debido principalmente a los altos precios alcanzados por el cobre, a la alta inversión extranjera y a su creciente apertura comercial e inserción en los mercados internacionales.

2 Según las estadísticas del Ministerio de Desarrollo Social y Familia, en el año 2000 un 26 \% de la población chilena era considerada pobre, mientras que en 2017 dicho porcentaje se redujo a un 8,6 \%, cifra equivalente a 1,5 millones de personas. 
Grindle, 2004). Incluso cuan robusta sea la institucionalidad que cobija a los gobiernos locales, provocará impactos positivos sobre la generación de capital social a nivel local (Warner, 2001).

Bajo este contexto, el presente estudio analiza el efecto del gasto público en educación per cápita, ejecutado por los gobiernos locales chilenos, sobre la desigualdad de ingresos a nivel comunal. La investigación se centró en cuatro medidas de gasto municipal en educación (gasto total, en personal, en inversión y tasa de inversión), mientras que la desigualdad fue cuantificada mediante los índices de Gini y Theil y los indicadores 10/10 y 20/20. El estudio longitudinal consideró un panel balanceado que incluye 316 comunas chilenas para el periodo 2006-2017, construido a partir de las seis últimas rondas de la Encuesta de Caracterización Socioeconómica Nacional (CASEN). Lo anterior permite estimar modelos probit de respuesta fraccional para el coeficiente de Gini y Tobit de efectos aleatorios para los restantes indicadores de desigualdad de ingresos. La evidencia encontrada indica que aumentos en el gasto en educación per cápita reducirían la desigualdad de ingresos, medida a través del índice de Theil, pero acentuarían la brecha de ingresos entre el decil/quintil más rico y el más pobre de la población. Además, el mayor gasto en personal educativo incrementaría el coeficiente de Gini y el ratio 10/10. Por otra parte, una mayor presencia de población indígena y rural exacerbaría la desigualdad comunal en Chile. Finalmente, la evidencia revela la existencia de una curva de Kuznets convexa para los valores extremos de la distribución de ingreso.

El resto del documento se organiza como sigue. La sección 2 aborda la literatura ligada al bienestar social, su relación con la distribución de ingresos y los principales determinantes de esta última. En la sección 3 se describe brevemente el sistema educacional chileno y su financiamiento, y se presenta la metodología empleada en la presente investigación, junto con una caracterización de los datos utilizados. La sección 4 expone los resultados y la discusión de sus alcances. La sección 5 concluye el documento.

\section{Revisión de la literatura}

\subsection{Bienestar social y desigualdad}

Las desigualdades sociales están hoy en el centro de la discusión mundial, especialmente en América Latina. Numerosos estudios ponen el foco en ella, suscitando debates de variada intensidad sobre sus causas, manifestaciones y consecuencias (Programa de las Naciones Unidas para el Desarrollo [PNUD], 2017). El estudio de tales desigualdades se relaciona con la economía del bienestar y la medición del bienestar social. Esta última implica evaluar las condiciones de vida de los individuos, lo que ha migrado desde considerar indicadores simples como el ingreso hacia otros indicadores compuestos que son más informativos y que incluyen variables adicionales, como la tasa de mortalidad, propensión a la morbilidad, nivel de escolaridad, estado de nutrición, participación política, entre otras.

Para la antigua economía del bienestar, vinculada con el utilitarismo clásico, una sociedad justa es aquella que ha logrado maximizar la suma de las utilidades individuales. Dado esto, el bienestar general de la sociedad aumenta en mayor proporción cuando se incrementa la utilidad del grupo menos favorecido (Pigou, 1932). Por ende, la función de bienestar social juega un rol clave en la determinación de la distribución óptima de ingresos, en la que el estado inicial de esta última no necesariamente constituye un óptimo de Pareto (Champernowne, 1953; Thurow, 1971).

Del estudio de la teoría del bienestar surgió una problemática central: su medición. Al respecto, el ingreso se ha utilizado habitualmente como una forma de medir la calidad de vida de la sociedad y la 
productividad de quienes lo perciben, constituyendo además una recompensa para quienes poseen mayores atributos (Lebergott, 1959; Sadka, 1976). En esta línea, Newhouse (1971) plantea que la educación o capacitación permite aumentar la productividad de trabajadores poco calificados, por lo que una fuerza laboral más educada y entrenada debería acceder a empleos mejor remunerados. En la literatura aún persiste la controversia sobre los alcances y limitaciones de utilizar el ingreso y el gasto corriente de los hogares como medidas adecuadas para evaluar la calidad de vida de un grupo familiar. Medina (1998) señala que cuando se utiliza el ingreso como medida de bienestar es muy probable que se presenten problemas en aquellas situaciones en las que las familias desahorran o se endeudan para poder adquirir bienes y servicios, distorsionando la medición del bienestar a través de este indicador.

\subsection{Determinantes de la desigualdad de ingresos}

El interés por dilucidar aquellos factores que explicarían cambios en la distribución del ingreso es gatillado por la hipótesis delineada por Kuznets (1955). Esta hipótesis plantea la existencia de una relación directa entre crecimiento económico y desigualdad de ingresos en las primeras etapas del desarrollo de una economía, que a partir de un umbral comienza a verse disminuida conforme el crecimiento avanza y esta presenta rasgos más propios de un sistema capitalista. Por ende, aquellos países que exhiben mayores niveles de crecimiento convergerían hacia una economía socialmente justa en el largo plazo (Aigner y Heins, 1967).

La dirección de la causalidad identificada por Kuznets ha sido puesta a prueba en la literatura. Para Copeland (1947) y Aaron y McGuire (1970), la política pública es un factor relevante para mejorar la distribución del ingreso, pues permite modificar sus patrones estructurales, lo que afecta favorablemente la producción agregada. Según Strassmann (1956) y Galor y Zeira (1993), el crecimiento económico no depende solo de la formación de capital y el progreso tecnológico, sino también de la creciente igualdad de ingresos, que elevaría la eficiencia marginal del capital. Un canal de transmisión alternativo fue identificado por Alesina y Perotti (1996), quienes señalan que una desigualdad de ingresos creciente genera efectos nocivos sobre la estabilidad política, reduciendo por esta vía el potencial de crecimiento de una economía.

En esta misma línea, la evidencia para América Latina indica que la relación entre crecimiento económico y distribución de ingresos tiene la forma de U invertida, sugerida por Kuznets. De acuerdo con Morley (2000), esta relación se ha vuelto más regresiva a través de los años en América Latina, debido a que el mayor crecimiento no ha mejorado la distribución de ingresos en la región. Ejemplo de ello es el caso chileno. Al respecto, Larrañaga (1999) estudió la dinámica existente entre el crecimiento económico sectorial y la distribución de ingresos de los hogares chilenos durante el periodo 1987-1996; encontró evidencia de aumentos en la desigualdad de ingresos a pesar de los niveles de crecimiento registrados para la economía chilena. En una línea similar, Álvarez et al. (2018) abordaron el efecto del denominado "súper ciclo" del precio del cobre, iniciado en 2003 por la alta demanda china por commodities, sobre la tasa de pobreza a nivel comunal. Los autores remarcaron que aquellas comunas más expuestas al shock positivo que afectó la actividad minera exhibieron la mayor reducción en los niveles de pobreza durante el periodo 1998-2013.

Otro de los aspectos macroeconómicos destacados en la literatura es el efecto que la inversión extranjera directa (IED) ejercería en los niveles de desigualdad. En este sentido, Te Velde (2003) remarcó que una de las principales causas de la mayor desigualdad de ingresos generada por la IED en 
América Latina obedece a que las empresas transnacionales tienden a localizarse en sectores de mano de obra altamente calificada, siendo estos los que absorben la mayoría de los beneficios de la IED. Por su parte, Suanes (2016) evidenció un efecto positivo de la IED sobre la desigualdad de ingresos para 13 economías de América Latina, especialmente en aquellas inversiones localizadas en los sectores de servicios e industria manufacturera.

Un tercer argumento macroeconómico que explicaría la distribución de ingresos se refiere a la carga impositiva o estructura tributaria de un país. Al respecto, Amarante y Jiménez (2016) demostraron para una muestra de países latinoamericanos ${ }^{3}$ que el impuesto a las altas rentas de los individuos tiene un efecto distributivo considerable, mejorando la recaudación fiscal y, por ende, aumentando la cantidad de recursos disponibles para programas sociales que permiten disminuir las tasas de pobreza y mejorar el bienestar social. Para Perotti (1992) el crecimiento económico es el resultado de la inversión privada en educación, por ende, la estructura redistributiva de impuestos determinará el acervo de capital humano, afectando la tasa de crecimiento de la economía en el largo plazo. De este modo, cualquier intervención gubernamental, sea de carácter regulatorio o de manejo de instrumentos de política económica, tiene consecuencias distributivas.

Por otra parte, la literatura destaca como argumentos que explican la desigualdad de ingresos la composición étnica de la población y la dicotomía urbano-rural del territorio. Respecto al primer argumento, Becker (1971) planteó que la discriminación, en conjunto con las desventajas iniciales del individuo, afectaría su desempeño en el mercado laboral y el potencial retorno de su inversión en capital humano. Desde la perspectiva empírica, la evidencia reportada por López (2016) revela que en las regiones del sur de Chile, que exhiben una mayor presencia de población indígena, la discriminación salarial también se incrementa, y parte de esta brecha podría estar explicada por el grado de conflicto que se ha instaurado en esta zona. Adicionalmente, Zurita y Dresdner (2009) analizaron la diferencia salarial entre la población mapuche y no indígena en Chile y se evidenció que existen diferencias significativas de dotaciones de capital humano a favor de la población no indígena. En cuanto a las diferencias territoriales, Cotte y Cotrino (2006) encontraron marcadas diferencias en los niveles de desigualdad entre las zonas rurales y urbanas de las comunas de Colombia, las que se explican principalmente debido a los bajos retornos de la educación que tendrían los individuos que habitan las zonas rurales.

\subsection{Rol del gasto público en educación en la reducción de inequidades}

Los énfasis de la política pública y la estructura del gasto social también juegan un rol en la distribución de ingresos. Según Mostajo (2000), el gasto social tiene impactos distributivos en el corto plazo mediante la provisión de salud, educación y transferencias monetarias, y también en el largo plazo, en el que la formación de capital humano aparece como principal instrumento para modificar la distribución de ingresos. En efecto, aumentos en los niveles medios de escolaridad ejercerían un efecto positivo sobre la distribución del ingreso, mientras que las disparidades en el logro educativo incrementarían las desigualdades (Tinbergen, 1957; Aigner \& Heins, 1967; Marin 8 Psacharopoulos, 1976; Winegarden, 1979; Beyer, 1999). De acuerdo con Ram (1989), el efecto de la escolaridad promedio de la fuerza laboral sobre los niveles de inequidad en países en desarrollo es marginal y de lenta entrega. En efecto, el autor remarca que una reducción de o,1 en el coeficiente de Gini de países menos desarrollados se manifestaría tras medio siglo de espera, ello debido a que la escolaridad promedio tarda al menos una 
década en exhibir un incremento de un año adicional. Empleando una muestra más amplia de países, De Gregorio y Lee (2002) confirmaron que la desigualdad de ingresos se ve incrementada ante una mayor desigualdad en la escolaridad promedio, entendiéndose esta última como la desviación estándar del máximo nivel educacional alcanzado por la población.

Dado lo anterior, una vía para incrementar la escolaridad promedio de la población es fortalecer el acceso de esta última a la educación pública de calidad en sus distintos niveles, a través de una expansión permanente del gasto público en educación. La interrogante que emerge es si este mayor gasto público realmente reduce los niveles de disparidad de ingreso o genera el efecto contrario. Al respecto, Sylwester (2002) encontró evidencia que avala la relación negativa entre el gasto público en educación como porcentaje del PIB y la variación en el coeficiente de Gini, empleando una muestra de 50 países desarrollados y emergentes para el periodo 1960-1990. En línea con lo reportado por Ram (1989), los resultados sugieren que, en promedio, un punto porcentual adicional de gasto en educación reduciría el coeficiente de Gini en un punto; no obstante, dicho efecto positivo sobre la desigualdad sería lento y tomaría alrededor de veinte años en materializarse.

A diferencia de estudios anteriores, Keller (2010) emplea diversas medidas de gasto público en educación según el nivel educativo al que se destina: tasa de cobertura, gasto público como porcentaje del PIB y gasto público por estudiante como porcentaje del PIB per cápita. Lo interesante es que la evidencia encontrada para los 77 países en desarrollo que consideró el estudio no es concluyente sobre el efecto negativo del gasto en educación sobre el coeficiente de Gini. En particular, los resultados indican que aumentos en la tasa de cobertura y el gasto en educación como porcentaje del PIB en educación primaria generan incrementos en el citado indicador, efecto que es exacerbado en países latinoamericanos y africanos. Por el contrario, solo un mayor gasto en educación terciaria como porcentaje del PIB reduciría la desigualdad, mientras que el gasto público por estudiante parece no alterar la distribución de ingresos al interior de países menos desarrollados.

A través de un análisis de metarregresión, Abdullah et al.(2015) trataron de dilucidar el verdadero efecto de la educación sobre la desigualdad, dada la contradictoria evidencia presente en la literatura. Tras la selección rigurosa de 64 estudios de un total de 2266 disponibles en las bases de datos JSTOR y EconLit, los autores concluyeron que la educación no generaría efecto alguno, en promedio, sobre la distribución de ingresos medida por el coeficiente de Gini. No obstante, la educación sí reduciría la desigualdad entre los grupos extremos de la distribución de ingresos, siendo la educación secundaria la que provocaría los mayores efectos redistributivos.

Finalmente, destaca el interés creciente de la literatura por abordar tanto aquellos canales de transmisión alternativos que condicionarían el impacto de la educación sobre la desigualdad (e. g., la disparidad en el acceso de la población a internet y las TIC) como redireccionar el foco hacia el efecto de la educación sobre la movilidad social intergeneracional en economías emergentes (Kudasheva, Kunitsa, Mukhamediyev, 2015; Yang 83 Qiu, 2016).

\section{Materiales y métodos}

\subsection{El sistema educacional chileno y su financiamiento}

El sistema educacional chileno comprende cuatro niveles de enseñanza: preescolar, primario, secundario y terciario. El primer nivel se extiende hasta los 5 años de vida de los estudiantes, el segundo nivel 
está entre los 6 y 13 años, mientras que el tercer nivel va desde los 14 hasta los 17 años. El último nivel se desarrolla durante la vida adulta.

En la enseñanza parvularia, básica y media, la administración de los establecimientos educacionales es ejercida por los gobiernos locales (municipios), corporaciones de administración delegada, prestadores privados que pueden optar o no a la subvención estatal, conocidos como establecimientos particularsubvencionado y particular-pagado, respectivamente.

En la educación terciaria, la oferta académica depende de centros de formación técnica (CFT), institutos profesionales (IP), universidades tradicionales pertenecientes al Consejo de Rectores de las Universidades Chilenas (CRUCH), que incluye instituciones estatales y corporaciones de derecho privado sin fines de lucro, y universidades privadas.

En vista de la coexistencia de actores públicos y privados en todos los niveles de enseñanza, el sistema educacional chileno se financia de manera mixta incluyendo el gasto de las familias. Respecto al rol de los municipios en el financiamiento de los tres primeros niveles de educación, estos pueden utilizar recursos propios para complementar aquellos recibidos desde el gobierno central a través del Ministerio de Educación (MINEDUC). Este gasto municipal en educación puede distribuirse principalmente entre los siguientes ítems: personal, compra de bienes y servicios, transferencias e inversión real (Marcel y Tokman, 2005).

\subsection{Datos}

Cabe recordar que el objetivo del presente estudio fue analizar el efecto del gasto público municipal per cápita en educación sobre la distribución de ingresos autónomos a nivel comunal en Chile. Para lograr dicho objetivo fue necesario contar con alguna medida de desigualdad en la distribución de ingresos, en la que el coeficiente de Gini es el indicador más utilizado para tal efecto. Este coeficiente se calcula a partir de la curva de Lorenz, siendo generalmente presentado en su forma estandarizada, con lo cual adquiere el valor máximo de 1, en el caso de máxima desigualdad, y o, en el caso de perfecta igualdad. Dentro de las desventajas de este indicador se encuentra que no es demasiado útil para estudiar brechas distributivas en la distribución de ingresos. Para ello existen ciertos indicadores locales tales como los ratios 10/10 y 20/20; el primero indica la razón entre el ingreso promedio de los deciles de mayores y menores ingresos, en tanto que el segundo captura la razón de ingresos entre el primer y último quintil. Adicionalmente, Contreras y Ruiz-Tagle (1997) destacaron como medidas de desigualdad complementarias los coeficientes de variación, de Atkinson y de Theil, que presentan características particulares. $^{4}$

Por consiguiente, el presente estudio empírico abordó la desigualdad de ingresos considerando cuatro de las medidas anteriores: los coeficientes de Gini y Theil y los ratios 10/10 y 20/20. Para calcular tales indicadores a nivel comunal, se utilizó el ingreso autónomo de los hogares chilenos, disponible en las diversas rondas de la Encuesta CASEN aplicadas entre los años 2006 y 2017. Este instrumento es elaborado por el Ministerio de Desarrollo Social y mide el estándar de vida de los hogares en Chile. La encuesta entrega un acabado perfil socioeconómico de los hogares, abordando ámbitos como vivienda, salud, educación y registrando incluso las distintas fuentes de ingresos percibidos por cada uno de sus miembros (ocupación principal, secundaria, subsidios monetarios, etc.).

4 De acuerdo con Contreras y Ruiz-Tagle (1997), los coeficientes de Atkinson y de Theil son más sensibles a los cambios en la parte baja de la distribución, mientras que el coeficiente de variación es más sensible a los cambios en su parte alta. 
Como se recalcó en la introducción, los niveles de desigualdad de ingresos en Chile han permanecido casi invariantes durante las últimas décadas. El estudio de este fenómeno se ha focalizado en la distribución del ingreso y la pobreza a nivel nacional y regional, pero con poca atención a nivel comunal (Agostini \& Brown, 2007).

Al centrar la atención en la desigualdad regional de ingresos durante el periodo de análisis, esta ha permanecido constante en algunas regiones de Chile (ver Gráfico 1). En particular, en el año 2006, la región Metropolitana lideró la desigualdad regional de ingresos con un coeficiente de Gini de o,539, seguida por las regiones de Arica y Parinacota (o,535), La Araucanía (0,534), del Biobío (o,522), Los Ríos $(0,516)$ y Los Lagos $(0,504)$, siendo estas cuatro últimas pertenecientes a la zona centro sur del país. En el año 2017, los coeficientes de desigualdad de ingresos disminuyeron levemente con respecto al año 2006, a excepción de la región de Arica y Parinacota, que exhibe una reducción del 20,5\%. Cabe destacar que la región Metropolitana sigue siendo la región más desigual de Chile, con un coeficiente de Gini de o,507. Le siguen La Araucanía (o,503), Los Ríos (o,487), Aysén (o,486), Los Lagos $(0,486)$ y Biobío $(0,48)$.

\section{Gráfico 1. Coeficiente de Gini según regiones de Chile, años 2006 y 2017}

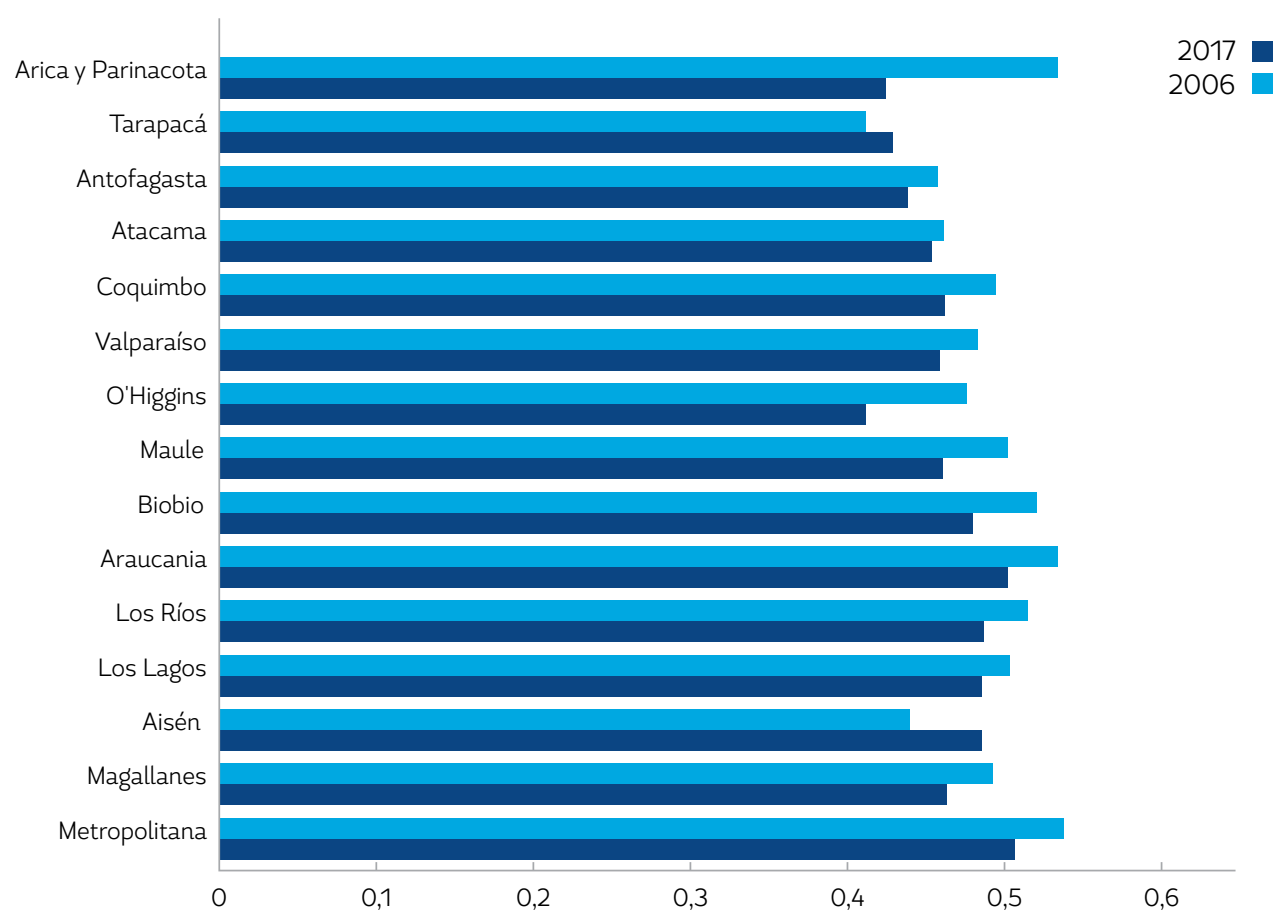

Fuente: elaboración propia a partir de la Encuesta CASEN.

En términos de desigualdad comunal, destaca la comuna de Galvarino, región de La Araucanía, que en el año 2006 registró el mayor nivel de desigualdad de ingresos en Chile, reportando un coeficiente de Gini de 0,729 y un índice de Theil de 1,641 (ver Tabla 1). Una explicación de los altos niveles de desigualdad de Galvarino podría ser que el decil más alto de la distribución percibe un ingreso 162,82 veces mayor que el decil de menores ingresos. En segundo lugar, la comuna de Sierra Gorda de la región de Antofagasta presenta un coeficiente de Gini de o,683, un índice de Theil de 1,506 y un indicador $10 / 10$ de 58 veces. 
Tal como se aprecia en la Tabla 1, las comunas con peor distribución del ingreso en el año 2017 , medida a través del coeficiente de Gini e índice de Theil, pertenecen a las regiones de Los Lagos y La Araucanía. Por consiguiente, tales territorios deberían ser el foco para la actual política social, identificando los principales factores de tales niveles de desigualdad.

Tabla 1. Comunas de Chile con mayor nivel de desigualdad de ingresos

\begin{tabular}{|c|c|c|c|c|c|c|c|}
\hline \multirow[t]{5}{*}{ Año } & Comuna & Región & Ranking & Gini & Theil & $10 / 10$ & $20 / 20$ \\
\hline & Galvarino & La Araucanía & 1 & 0,729 & 1,641 & 162,82 & 62,07 \\
\hline & Sierra Gorda & Antofagasta & 2 & 0,683 & 1,506 & 57,75 & 20,98 \\
\hline & Lo Barnechea & Metropolitana & 3 & 0,670 & 0,925 & 90,92 & 34,49 \\
\hline & Colchane & Tarapacá & 4 & 0,664 & 1,038 & 30,82 & 21,80 \\
\hline \multirow[t]{10}{*}{2006} & Santo Domingo & Valparaíso & 5 & 0,656 & 0,889 & 41,37 & 18,13 \\
\hline & Pucón & La Araucanía & 6 & 0,639 & 1,328 & 66,69 & 25,39 \\
\hline & Primavera & Magallanes & 7 & 0,637 & 0,810 & 22,08 & 11,35 \\
\hline & San Carlos* & Biobío & 8 & 0,627 & 0,982 & 61,97 & 23,32 \\
\hline & San Pablo & Los Lagos & 9 & 0,611 & 0,893 & 37,94 & 18,22 \\
\hline & Ninhue* & Biobío & 10 & 0,610 & 0,833 & 56,61 & 20,14 \\
\hline & Chonchi & Los Lagos & 1 & 0,649 & 1,511 & 400,19 & 50,86 \\
\hline & Curarrehue & La Araucanía & 2 & 0,622 & 0,920 & 85,50 & 23,97 \\
\hline & Colina & Metropolitana & 3 & 0,619 & 0,809 & 46,13 & 21,82 \\
\hline & Cabildo & Valparaíso & 4 & 0,610 & 1,097 & 141,97 & 44,39 \\
\hline \multirow[t]{6}{*}{2017} & Lo Barnechea & Metropolitana & 5 & 0,605 & 0,674 & 69,62 & 20,79 \\
\hline & La Serena & Coquimbo & 6 & 0,598 & 1,428 & 84,73 & 22,10 \\
\hline & $\begin{array}{l}\text { San Juan de la } \\
\text { Costa }\end{array}$ & Los Lagos & 7 & 0,592 & 0,640 & 15,88 & 17,59 \\
\hline & Vichuquén & Maule & 8 & 0,590 & 1,023 & 103,11 & 26,29 \\
\hline & $\begin{array}{l}\text { San Pedro de } \\
\text { la Paz }\end{array}$ & Biobío & 9 & 0,583 & 0,644 & 36,37 & 15,15 \\
\hline & Antuco & Biobío & 10 & 0,582 & 0,704 & 32,50 & 17,45 \\
\hline
\end{tabular}

Nota: información según coeficiente de Gini e indicadores complementarios de inequidad, años 2006 y 2017. Las comunas destacadas con asterisco pertenecen en la actualidad a la región de Ñuble, la cual entró en vigor el 6 de septiembre del 2018.

Fuente: elaboración propia a partir de la Encuesta CASEN.

\subsection{Estrategia empírica}

Para estimar el efecto del gasto en educación per cápita sobre la distribución de ingresos para las comunas de Chile, el presente estudio utilizó el ingreso autónomo del hogar para construir los distintos indicadores de desigualdad. ${ }^{5}$ Esta variable, disponible en las diversas rondas de la Encuesta CASEN, representa la suma de los ingresos percibidos por los distintos miembros de un hogar, excluyendo los subsidios monetarios y alquileres imputados. ${ }^{6}$ Teniendo en cuenta que la Encuesta CASEN aún posee problemas para representar zonas geográficas extremas, y que el Ministerio de Desarrollo Social y Familia reconoce a nivel comunal un error muestral mayor que a nivel regional y nacional, no es posible considerar en el presente estudio las 346 comunas que comprenden el territorio chileno.

5 De Gregorio y Lee (2002) destacan que el cálculo de indicadores de desigualdad a nivel de hogares suele entregar estimaciones menores del coeficiente de Gini respecto de aquellas que utilizan datos a nivel individual.

6 La Encuesta CASEN identifica el ingreso autónomo del hogar a través de las variables yauthaj durante las rondas 2006, 2009 y 2011, y yautcorh en las versiones 2013, 2015 y 2017 del instrumento. 
Por consiguiente, el presente estudio longitudinal consideró un panel balanceado para el periodo 2006-2017 de 316 comunas para estimar el coeficiente de Gini y el índice de Theil, 226 comunas para el índice 10/10 y 311 municipios para el índice 20/20.

Adicionalmente, el estudio empírico reconoció las características de la variable dependiente, al momento de seleccionar el método de estimación. En primer lugar, dado que los valores del coeficiente de Gini se encuentran entre o y 1, este se modeló a través del enfoque probit de respuesta fraccional desarrollado por Papke y Wooldridge (1996, 2008). Bajo este enfoque, la heterogeneidad no observada, $c_{i}$, se correlaciona con el promedio de aquellos regresores que varían en el tiempo de la forma sugerida por Mundlak (1978) y Chamberlain (1980). Por lo tanto, el modelo probit de respuesta fraccional para el coeficiente de Gini se especifica del siguiente modo:

$$
\begin{gathered}
E\left[\operatorname{gini}_{i t} \mid X_{i t}, c_{i}\right]=\Phi\left(X_{i t} \beta+c_{i}\right) \\
X_{i t} \beta=\alpha \text { geducper }_{i t-j}+z_{i t} \gamma+\varepsilon_{i t} ; i=1, \ldots, N ; t=1, \ldots, T ; j=1,2 \\
c_{i}=\psi+\bar{G}_{i} \xi+a_{i}
\end{gathered}
$$

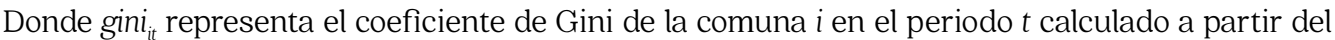
ingreso autónomo del hogar, mientras que $\Phi(\cdot)$ es la función de densidad acumulada de la distribución normal estándar. Por su parte, el vector geducper ${ }_{i t-j}$ captura el gasto en educación per cápita (i.e., por estudiante) que realizan las municipalidades en los establecimientos educacionales que se encuentran bajo su administración, es decir, se excluyen las escuelas, colegios y liceos particular-subvencionados y particular-pagados. Este gasto, medido en miles de pesos chilenos, comprende cuatro medidas: gasto total devengado (geducper_total), gasto en personal de educación (geducper_personal), gasto en inversión real (geducper_inversion) y el porcentaje del gasto destinado a inversión (geducper_tasainversion). Adicionalmente, se consideró adecuado capturar el eventual efecto rezagado que podría provocar el gasto en educación sobre la distribución de ingresos, incluyendo especificaciones donde la variable geducper $_{i t-j}$ fue rezagada en uno, dos y tres periodos $(j=1, j=2$ y $j=3$, respectivamente). Todas estas medidas fueron deflactadas por el índice de precios al consumidor base 2018, reportado por el Instituto Nacional de Estadísticas de Chile. La información a nivel comunal de gasto municipal en educación y matrícula en establecimientos municipales de educación fue extraída del Sistema Nacional de Información Municipal (SINIM). ${ }^{7}$

La modelación empírica también incluyó un vector de variables de control, $z_{i t}$, el cual considera el logaritmo natural del ingreso autónomo per cápita del hogar (Inypercap), en miles de pesos chilenos de 2008, y su cuadrado (Inypercap2); el porcentaje de población indígena (etnia), y el porcentaje de la población comunal que habita en la zona rural (ruralidad), siendo todas estas variables calculadas a partir de la Encuesta CASEN. Con el objeto de capturar el efecto de concentración o centralización en la toma de decisiones que ejercería la capital provincial/regional al interior de una región, el vector incluye la distancia en kilómetros desde el centroide de la comuna $i$ hasta el centroide de su respectiva capital regional (dist_capreg) y una variable dicotómica (capitalprov) que toma el valor 1 si la comuna $i$ es la capital de la provincia en que está inserta y o en caso contrario. ${ }^{8}$

7 Esta información se encuentra disponible públicamente en el sitio web dispuesto para ello por la Subsecretaría de Desarrollo Regional y Administrativo (SUBDERE): http://datos.sinim.gov.cl/datos_municipales.php

8 Hasta el año 2016, Chile poseía 54 capitales provinciales, siendo 51 de ellas incluidas en la muestra del presente estudio. 
Cabe destacar que durante gran parte del periodo analizado, Chile experimentó un shock positivo en el precio del cobre, principal commodity de su canasta exportadora. Tal como lo remarcan Álvarez et al. (2018), lo anterior trajo consigo mayores tasas de crecimiento y una reducción en los indicadores de pobreza a nivel nacional y, en especial, en aquellas regiones y comunas con mayor presencia de la actividad minera (e.g., región de Antofagasta). En línea con la metodología implementada por los autores, se adicionaron como controles el porcentaje del empleo comunal generado por el sector minero (empleomineria) y variables dicotómicas por año.

En último término, $\varepsilon_{i t}$ es el error idiosincrático y la heterogeneidad no observada $c_{i}$ se distribuye $c_{i} \mid X \sim N\left(\psi+\bar{G}_{i} \xi, \sigma_{a}^{2}\right)$, donde $\bar{G}_{i}$ es el promedio en el tiempo de todos los regresores que varían en el tiempo.

Luego de ser estimado el modelo anterior, se procedió al cómputo de los efectos parciales promedio, siguiendo a Papke y Wooldridge (2008). Esto implica que si el regresor $x_{j}$ es continuo, entonces su efecto parcial promedio (o APE en inglés por average partial effect) viene dado por:

$$
\frac{\partial E\left[\operatorname{gini}_{i t} \mid X_{i t}, c_{i}\right]}{\partial x_{j}}=\frac{1}{N T} \sum_{i=1}^{N} \sum_{t=1}^{T} \hat{\beta}_{j} \phi\left(\hat{\psi}+X_{i t} \beta+\bar{G}_{i} \xi\right)
$$

Por otro lado, en el caso de que el regresor $x_{j}$ sea una variable dicotómica, entonces su APE se define como:

$$
\frac{\partial E\left[\text { gini }_{i t} \mid X_{i p} c_{i}\right]}{\partial x_{j}}=\frac{1}{N T} \sum_{i=1}^{N} \sum_{t=1}^{T}\left[\Phi\left(\hat{\psi}+X_{i t} \hat{\beta}+\bar{G}_{i} \hat{\xi}\right)\left|x_{j=1}-\Phi\left(\hat{\psi}+X_{i t} \hat{\beta}+\bar{G}_{i} \hat{\xi}\right)\right| x_{j=o}\right]
$$

Donde $\phi(\cdot)$ y $\Phi(\cdot)$ son las funciones de densidad y de densidad acumulada de una distribución de probabilidad normal estándar, respectivamente, mientras que el acento circunflejo denota valores estimados.

En segundo lugar, el índice de Theil y los ratios 10/10 y 20/20 se modelaron a través de un modelo Tobit de efectos aleatorios, debido a que estos tres indicadores son variables continuas pero truncadas (Wooldridge, 2002). Por ende, la estimación del índice de Theil, theil $l_{i t}$ y de los ratios 10/10 y 20/20, $r^{k}{ }^{k}$, se realizó mediante las siguientes especificaciones empíricas:

$$
\begin{gathered}
\text { theil }_{i t}=\left\{\begin{array}{cc}
0 & \text { Si } y^{*} \leq 0 \\
X_{i t} \beta & \text { Si } y^{*}>0
\end{array}\right. \\
r_{i t}^{k}=\left\{\begin{array}{cc}
1 & \text { Si } y^{*} \leq 1 \\
X_{i t} \beta & \text { Si } y^{*}>1
\end{array} ; k=10 / 10,20 / 10\right.
\end{gathered}
$$




$$
X_{i t} \beta=\text { ageducper }_{i t-j}+z_{i t} \gamma+\mathrm{u}_{i t} ; i=1, \ldots, N ; t=1, \ldots, T ; j=1,2
$$

Donde $y^{*}$ es una variable latente no observada y $c_{i}$ es la heterogeneidad no observada que se asume independiente del error idiosincrático $u_{i t}$, los que se distribuyen $c_{i} \mid X \sim N\left(0, \sigma_{c}^{2}\right)$ y $u_{i t} \mid X, c_{i} \sim N\left(0, \sigma_{u}^{2}\right)$.

De manera alternativa, los APE para el modelo Tobit de efectos aleatorios fueron calculados siguiendo a Cong (2000). Así, si el regresor $x_{j}$ es continuo, entonces su APE se obtiene mediante la siguiente expresión:

$$
\frac{\partial E\left[y_{i t} \mid a<y_{i t}<b\right]}{\partial x_{j}}=\frac{1}{N T} \sum^{N}{ }_{i=1} \sum_{t=1}^{T} \hat{\beta}_{j}\left[1-\left(\frac{\delta_{a} \phi\left(\delta_{a}\right)-\delta_{b} \phi\left(\delta_{b}\right)}{\Phi\left(\delta_{a}\right)-\Phi\left(\delta_{b}\right)}\right)-\left(\frac{\phi\left(\delta_{a}\right)-\phi\left(\delta_{b}\right)}{\Phi\left(\delta_{a}\right)-\Phi\left(\delta_{b}\right)}\right)^{2}\right]
$$

Donde $y_{i t}$ es la variable dependiente truncada, $\phi(\cdot)$ y $\Phi(\cdot)$ representan nuevamente las funciones de densidad y de densidad acumulada de una distribución de probabilidad normal estándar, respectivamente, y el acento circunflejo denota el valor estimado del parámetro $\beta_{j}$. Los escalares a y $b$ son los valores extremos en los que se encuentra truncada la variable dependiente; es decir, a es 0 para el índice de Theil y 1 para los ratios 10/10 y 20/20, mientras que el escalar $b$ es igual infinito para el índice de Theil y los ratios 10/10 y 20/20. Adicionalmente, $\delta_{a}$ se define como $\left(a-X_{i t} \beta\right) / \sigma$ y $\delta_{b}$ como $\left(b-X_{\text {it }} \beta\right) / \sigma$, donde $\sigma=\sqrt{\sigma_{c}^{2}+\sigma_{u}^{2}}$.

Finalmente, y a modo de información complementaria, la Tabla 2 presenta las principales estadísticas descriptivas de las variables dependientes y regresores utilizados en las especificaciones empíricas descritas.

\begin{tabular}{|c|c|c|c|c|c|}
\hline & Obs. & Media & $\begin{array}{l}\text { Desviación } \\
\text { estándar }\end{array}$ & Mínimo & Máximo \\
\hline \multicolumn{6}{|l|}{ Variables dependientes: } \\
\hline Coeficiente de Gini (ginit) & 1896 & 0,450 & 0,06 & 0,23 & 0,84 \\
\hline Índice de Theil (theilit) & 1896 & 0.389 & 0,17 & 0,11 & 2,61 \\
\hline Índice 10/10 $\left(r_{t i}^{10 / 0}\right)$ & 1356 & 32.041 & 29,58 & 3,59 & 480,62 \\
\hline Indice 20/20 $\left(r_{t}^{20 / 20}\right)$ & 1866 & 11,091 & 8,14 & 2,57 & 206,37 \\
\hline \multicolumn{6}{|l|}{ Gasto en educación municipal per cápita: } \\
\hline Total devengado (geducper_total) & 1896 & 1658,227 & 695,79 & 176,56 & 9915,55 \\
\hline Personal de educación (geducper_personal) & 1896 & 1338,707 & 533,92 & 147,91 & 6342,91 \\
\hline Inversión real (geducper_inversión) & 1896 & 20,315 & 60,53 & 0 & 1207,23 \\
\hline Inversión sobre gasto total (geductasainversión) & 1896 & 0,013 & 0,033 & 0 & 0,384 \\
\hline \multicolumn{6}{|l|}{ Variables de control: } \\
\hline Ln Ingreso per cápita del hogar (Lnypercap) & 1896 & 5,353 & 0,40 & 4,41 & 7,51 \\
\hline Ln Inoreso per cápita del hogar al cuadrado (Inypercap2) & 1896 & 28,810 & 4,53 & 19,42 & 56,47 \\
\hline Porcentaje población indígena (etnia) & 1896 & 0,124 & 0,17 & 0 & 0,96 \\
\hline Porcentaje población rural (ruralidad) & 1896 & 0,356 & 0,28 & 0 & 1 \\
\hline Distancia hacia capital regional (dist_capreg) & 1896 & 68,458 & 53,60 & 0 & 352,29 \\
\hline Capital provincial (capitalprov) & 1896 & 0,161 & 0,37 & 0 & 1 \\
\hline Porcentaje empleo sector minero (empleomineria) & 1896 & 0,029 & 0,06 & 0 & 0,537 \\
\hline
\end{tabular}

Tabla 2. Estadística descriptiva de las variables consideradas en el estudio

Fuente: elaboración propia.

9 Note que si el escalar b tiende a infinito, entonces $\Phi\left(\boldsymbol{\delta}_{b}\right) \rightarrow 1$ y $\phi\left(\boldsymbol{\delta}_{b}\right) \rightarrow 0$. 


\section{Principales resultados y discusión}

En esta sección se presentan y discuten los resultados obtenidos tras la estimación de los modelos empíricos descritos en el apartado anterior, haciendo la distinción entre aquellos indicadores que engloban la totalidad de la distribución de ingresos y los que se focalizan en sus valores extremos, considerando el gasto en educación per cápita contemporáneo a las mediciones de desigualdad.

En primer lugar, la Tabla 3 reporta los resultados tras la estimación del modelo base para el coeficiente de Gini e índice de Theil. La evidencia encontrada sugiere, con un nivel de confianza del $90 \%$, que el gasto en educación per cápita reduciría la desigualdad de ingresos comunal en Chile solo si esta es medida a través del índice de Theil (columna 4, Tabla 3). Dada la magnitud del APE estimado, se requerirían 1,5 millones de pesos adicionales por estudiante para lograr una disminución de 3 centésimas en el índice de Theil. Cabe destacar que esta cifra, equivalente en 2018 a 3780 dólares internacionales (USD PPP), es muy cercana al incremento promedio experimentado por el gasto municipal per cápita durante el periodo 2006-2017. Lo anterior confirmaría lo destacado por Ram (1989) y Sylwester (2002) respecto a la lentitud del proceso de ajuste de la distribución de ingresos hacia una sociedad más equitativa tras aumentos en el gasto público destinado a educación.

Tras la desagregación del gasto municipal en educación, los resultados indican, con un nivel de significancia del $10 \%$, que los recursos destinados al personal del sector educacional municipal aumentarían el coeficiente de Gini en un punto por cada o,5 millones de pesos (126o USD PPP) adicionales (columnas 2 y 3, Tabla 3). Lo anterior podría explicarse por la caída dramática en la matrícula de establecimientos municipales de educación primaria y secundaria experimentada en la última década y la rigidez para ajustar la planta docente a nivel municipal. Estos establecimientos dependientes de los gobiernos locales exhiben, en promedio, el peor rendimiento en las pruebas estandarizadas aplicadas en Chile para medir la calidad del proceso enseñanza-aprendizaje. Esto generaría efectos nocivos sobre la probabilidad de ingresar a la educación superior de quienes se enrolan en la educación municipal, coartando la movilidad social de la población de menores ingresos y acentuando los niveles de desigualdad.

Por otra parte, el gasto municipal en inversión por estudiante y la participación de la inversión en el gasto total en educación parecen no afectar los niveles de desigualdad comunal medidos por los indicadores Gini y Theil. Este resultado debe ser mirado con cautela, debido a que una alta proporción de las comunas reportan no haber realizado inversión alguna en educación durante el periodo analizado. De acuerdo con la información del SINIM, en 2006, el 23,2 \% de las comunas no destinó recursos a educación, mientras que en 2017 esta cifra se elevó a 61,2 \%, lo que podría ser indicio de un subreporte de información municipal, ya que gran parte de los gobiernos locales declaran la educación como un objetivo relevante en su instrumento de planeación estratégica conocido como PLADECO. 
Tabla 3. Efectos parciales promedio (APE) tras la estimación probit de respuesta fraccional y Tobit de efectos aleatorios, variante gasto en educación contemporáneo

\begin{tabular}{|c|c|c|c|c|c|c|}
\hline \multirow{2}{*}{ Variable dependiente: } & \multicolumn{3}{|c|}{ Gini } & \multicolumn{3}{|c|}{ Theil } \\
\hline & (1) & (2) & (3) & (4) & (5) & (6) \\
\hline \multirow[t]{2}{*}{ geducper_total } & 0,0000 & & & $-0,00002^{*}$ & & \\
\hline & $(0,000)$ & & & $(0,000)$ & & \\
\hline \multirow[t]{2}{*}{ geducper_personal } & & $0,00002^{*}$ & $0,00002^{*}$ & & $-0,00001$ & $-0,00001$ \\
\hline & & $(0,000)$ & $(0,000)$ & & $(0,000)$ & $(0,000)$ \\
\hline \multirow[t]{2}{*}{ geducper_inversion } & & 0,00001 & & & $-0,0001$ & \\
\hline & & $(0,000)$ & & & $(0,000)$ & \\
\hline \multirow[t]{2}{*}{ geductasainversion } & & & 0,0286 & & & $-0,0796$ \\
\hline & & & $(0,037)$ & & & $(0,107)$ \\
\hline \multirow[t]{2}{*}{ Inypercap } & 0,1215 & 0,1118 & 0,1118 & $0,7981^{* * *}$ & $0,8029^{* * *}$ & $0,8044^{* * *}$ \\
\hline & $(0,118)$ & $(0,116)$ & $(0,116)$ & $(0,145)$ & $(0,145)$ & $(0,145)$ \\
\hline \multirow[t]{2}{*}{ Inypercap2 } & 0,0006 & 0,0015 & 0,0015 & $-0,0545^{* * *}$ & $-0,0551^{* * *}$ & $-0,0552^{* * *}$ \\
\hline & $(0,011)$ & $(0,011)$ & $(0,011)$ & $(0,013)$ & $(0,013)$ & $(0,013)$ \\
\hline \multirow[t]{2}{*}{ etnia } & $0,0968 * * *$ & $0,0958^{* * *}$ & $0,0957^{* * *}$ & $0,1657^{* * *}$ & $0,1601^{* * *}$ & $0,1560^{* * *}$ \\
\hline & $(0,028)$ & $(0,028)$ & $(0,028)$ & $(0,029)$ & $(0,029)$ & $(0,029)$ \\
\hline \multirow[t]{2}{*}{ ruralidad } & 0,0366 & $0,0372^{*}$ & $0,0374^{*}$ & $0,1166^{* * *}$ & $0,1121^{* * *}$ & $0,1126^{* * *}$ \\
\hline & $(0,023)$ & $(0,022)$ & $(0,022)$ & $(0,021)$ & $(0,021)$ & $(0,021)$ \\
\hline \multirow[t]{2}{*}{ distancia_capitalreg } & 0,0001 & 0,0001 & 0,0001 & 0,0002 & 0,0002 & 0,0002 \\
\hline & $(0,000)$ & $(0,000)$ & $(0,000)$ & $(0,000)$ & $(0,000)$ & $(0,000)$ \\
\hline \multirow[t]{2}{*}{ capitalprov } & $0,0118^{* *}$ & $0,0117 *$ & $0,0120^{* *}$ & $-0,0088$ & $-0,0084$ & $-0,0081$ \\
\hline & $(0,005)$ & $(0,006)$ & $(0,006)$ & $(0,014)$ & $(0,014)$ & $(0,014)$ \\
\hline \multirow[t]{2}{*}{ empleomineria } & $-0,0088$ & $-0,0095$ & $-0,0098$ & $-0,3431 * * *$ & $-0,3412^{* * *}$ & $-0,3412^{* * *}$ \\
\hline & $(0,048)$ & $(0,048)$ & $(0,048)$ & $(0,077)$ & $(0,077)$ & $(0,077)$ \\
\hline Observaciones & 1896 & 1896 & 1896 & 1896 & 1896 & 1896 \\
\hline N.o de comunas & 316 & 316 & 316 & 316 & 316 & 316 \\
\hline Test estadístico de Wald & 491,3 & 498,8 & 509,1 & 383,9 & 383 & 382,5 \\
\hline
\end{tabular}

Nota: se incluyen dummies anuales no reportadas. Errores estándar entre paréntesis. ${ }^{*} p<0,1,{ }^{* *} p<0,05,{ }^{* * *} p<0,01$. Fuente: elaboración propia. 
En relación con las variables de control, es posible remarcar lo siguiente. Primero, los resultados confirman la existencia de la curva de Kuznets (i.e., U invertida) en la medición de desigualdad a través del índice de Theil con un nivel de significancia del $1 \%$ (columnas 4-6, Tabla 3); siendo este resultado coherente con lo reportado por Ram (1989), De Gregorio y Lee (2002) y Keller (2010).

Segundo, las estimaciones sugieren que la composición étnica de la población es un determinante estadísticamente significativo al $1 \%$ que influye positivamente la desigualdad de ingresos en Chile cuantificada mediante los indicadores Gini y Theil (columnas 1-6, Tabla 3). En efecto, el APE estimado para la variable etnia da cuenta de mayores inequidades en comunas donde existe una alta concentración de personas pertenecientes a pueblos originarios. De acuerdo con el Censo de Población y Vivienda aplicado en Chile durante el año 2017, la población indígena se encuentra principalmente en la regiones norteñas de Arica y Parinacota (36\%) y Tarapacá (25\%), y en la zona sur en la región de la Araucanía (34\%) y en las comunas de Cañete (36\%), Contulmo (30\%), Tirúa (71\%) y Alto Biobío (86 \%), pertenecientes a la región del Biobío (Instituto Nacional de Estadísticas, 2018). Por ende, tales zonas geográficas deberían ser el foco de la política pública orientada a reducir las disparidades en la distribución de ingresos que enfrentan en Chile las etnias quechua, aymara y mapuche.

Tercero, el grado de ruralidad de una comuna también influye positivamente en la desigualdad de ingresos, siendo esta relación más robusta para el índice de Theil (columnas 2-6, Tabla 3). En este sentido, si se consideran solo las comunas incluidas en esta investigación, las regiones con mayor índice de ruralidad son Los Lagos $(0,53)$, Tarapacá $(0,50)$ y Maule $(0,49)$.

Cuarto, los resultados asociados al coeficiente de Gini indican, con un nivel de significancia del $5 \%$, que este indicador es superior en comunas que poseen el rango de capital provincial (columnas 1-3, Tabla 3), mientras que la distancia hacia la capital regional, centro del poder regional y de la toma de decisiones, parece no influir en la desigualdad de ingresos, medida por el coeficiente de Gini o Theil, lo que constituiría un indicio de descentralización intrarregional mayor al percibido por la población.

Quinto, aquellas comunas más expuestas al súper ciclo del precio del cobre sí se beneficiaron en reducir la inequidad de ingresos cuantificada por el índice de Theil (columnas 4-6, Tabla 3). Es decir, municipios donde el sector minero contribuye en mayor medida al empleo comunal experimentaron una mayor reducción de la desigualdad durante el periodo 2006-2017. Este resultado es robusto con un nivel de significancia del 1 \% y alineado con lo encontrado por Álvarez et al. (2018).

La Tabla 4 exhibe los resultados tras la estimación del modelo base para aquellas medidas de desigualdad enfocadas en los extremos de la distribución de ingresos, es decir, los ratios 10/10 y 20/20. La evidencia indica, con un nivel de significancia del $5 \%$, que un mayor gasto en educación por estudiante deteriora los niveles de equidad, acrecentando la distancia entre el ingreso promedio de quienes pertenecen a los deciles extremos de la distribución de ingresos comunal (columna 1, Tabla 4). Dada la magnitud del coeficiente estimado, el incremento promedio del gasto en educación per cápita observado durante el periodo 20062017 (i.e., 1,5 millones de pesos de 2018), habría incrementado en 4,95 veces el ratio 10/10 a nivel comunal.

De igual modo, el gasto en personal por estudiante también genera un impacto positivo y significativo al $5 \%$ en la desigualdad de ingresos entre el decil más rico y el más pobre a nivel comunal. Adicionalmente, los parámetros asociados a la inversión por estudiante y la participación de la inversión en el gasto total en educación son negativos para los ratios 10/10 y 20/20; sin embargo, estos no son estadísticamente significativos al $10 \%$, por lo que no generarían impactos en reducir la desigualdad comunal. 
Tabla 4. Efectos parciales promedio (APE) tras la estimación Tobit de efectos aleatorios, variante gasto en educación contemporáneo

\begin{tabular}{|c|c|c|c|c|c|c|}
\hline \multirow{2}{*}{ Variable dependiente: } & \multicolumn{3}{|c|}{$10 / 10$} & \multicolumn{3}{|c|}{$20 / 20$} \\
\hline & (1) & (2) & (3) & (4) & (5) & (6) \\
\hline \multirow[t]{2}{*}{ geducper_total } & $0,0033^{* *}$ & & & 0,0002 & & \\
\hline & $(0,002)$ & & & $(0,000)$ & & \\
\hline \multirow[t]{2}{*}{ geducper_personal } & & $0,0036^{* *}$ & $0,0035^{* *}$ & & 0,0002 & 0,0001 \\
\hline & & $(0,002)$ & $(0,002)$ & & $(0,000)$ & $(0,000)$ \\
\hline \multirow[t]{2}{*}{ geducper_inversion } & & $-0,0069$ & & & $-0,0008$ & \\
\hline & & $(0,010)$ & & & $(0,002)$ & \\
\hline \multirow[t]{2}{*}{ geductasainversion } & & & $-5,1030$ & & & $-1,9319$ \\
\hline & & & $(15,802)$ & & & $(3,722)$ \\
\hline \multirow[t]{2}{*}{ Inypercap } & $-46,5477^{*}$ & $-49,0337^{*}$ & $-48,5467^{*}$ & $-64,9614^{* * *}$ & $-65,0667^{* * *}$ & $-65,0428^{* * *}$ \\
\hline & $(27,911)$ & $(27,884)$ & $(27,900)$ & $(4,979)$ & $(4,978)$ & $(4,979)$ \\
\hline \multirow[t]{2}{*}{ Inypercap2 } & $6,1405^{* *}$ & $6,3830^{* *}$ & $6,3348^{* *}$ & $6,4458^{* * *}$ & $6,4571^{* * *}$ & $6,4552^{* * *}$ \\
\hline & $(2,493)$ & $(2,490)$ & $(2,491)$ & $(0,439)$ & $(0,439)$ & $(0,439)$ \\
\hline \multirow[t]{2}{*}{ etnia } & $-2,7060$ & $-2,3648$ & $-2,2834$ & 0,6444 & 0,6930 & 0,6894 \\
\hline & $(3,620)$ & $(3,565)$ & $(3,567)$ & $(0,816)$ & $(0,813)$ & $(0,813)$ \\
\hline \multirow[t]{2}{*}{ ruralidad } & $9,7039^{* * *}$ & $9.9682^{* * *}$ & $9,9604^{* * *}$ & $1,5550^{* *}$ & $1,5841^{* * *}$ & $1,5891^{* * *}$ \\
\hline & $(2,751)$ & $(2,730)$ & $(2,734)$ & $(0,617)$ & $(0,614)$ & $(0,614)$ \\
\hline \multirow[t]{2}{*}{ distancia_capitalreg } & $0,0203^{*}$ & 0,0199* & $0,0202^{*}$ & $0,0069^{* *}$ & $0,0068^{* *}$ & $0,0068^{* *}$ \\
\hline & $(0,011)$ & $(0,011)$ & $(0,011)$ & $(0,003)$ & $(0,003)$ & $(0,003)$ \\
\hline \multirow[t]{2}{*}{ capitalprov } & $-2,2419^{*}$ & $-2,2343^{*}$ & $-2,2175^{*}$ & $-0,7826^{* *}$ & $-0,7842^{* *}$ & $-0,7776^{* *}$ \\
\hline & $(1,322)$ & $(1,320)$ & $(1,323)$ & $(0,364)$ & $(0,364)$ & $(0,365)$ \\
\hline \multirow[t]{2}{*}{ empleomineria } & $-31,5606^{* * *}$ & $-32,5337^{* * *}$ & $-32,5107^{* * *}$ & $-9,4792^{* * *}$ & $-9,5116^{* * *}$ & $-9,5206^{* * *}$ \\
\hline & $(9,287)$ & $(9,224)$ & $(9,236)$ & $(2,181)$ & $(2,181)$ & $(2,182)$ \\
\hline Observaciones & 1356 & 1356 & 1356 & 1866 & 1866 & 1866 \\
\hline N.o de comunas & 226 & 226 & 226 & 311 & 311 & 311 \\
\hline Test estadístico de Wald & 266 & 266,5 & 265,7 & 744,1 & 744,4 & 744,2 \\
\hline
\end{tabular}

Nota: se incluyen dummies anuales no reportadas. Errores estándar entre paréntesis. ${ }^{*} p<0,1,{ }^{* *} p<0,05,{ }^{* * *} p<0,01$

Fuente: elaboración propia. 
Con relación a las variables de control, se destacan los siguientes seis puntos. Primero, la evidencia revela la existencia de una curva de Kuznets convexa (i.e., U no invertida) para la desigualdad de ingresos medida por los ratios 10/10 y 20/20 (columnas 1-6, Tabla 4). Esto sería señal de un fenómeno preocupante en términos de desigualdad, ya que esta última sería creciente entre los grupos extremos de la distribución de ingresos, a pesar de haber experimentado un ciclo positivo en los términos de intercambio gatillado por el alto precio del principal commodity exportado por Chile.

Segundo, las estimaciones además confirman la importancia de la ruralidad para explicar la desigualdad de ingresos entre los valores extremos de la distribución. De acuerdo con los APE reportados en la Tabla 4, un municipio totalmente rural exhibiría, en promedio, un indicador $20 / 20$ de 1,56 veces superior al de una comuna urbana, mientras que la diferencia de ingresos entre el decil más rico y el más pobre se elevaría a 9,88 veces solo por esta condición geográfica. Cabe agregar que los municipios rurales en Chile enfrentarían un fenómeno mucho más complejo en términos de desarrollo, que no se limita a la inequidad de ingresos. De acuerdo con el censo 2017, las localidades rurales presentan un menor acceso al agua potable y deficiencias en la materialidad de las viviendas, lo que evidencia un detrimento en la calidad de vida de sus habitantes.

Tercero, las estimaciones sugieren que la distancia hacia la capital regional tendría un impacto positivo sobre la desigualdad de ingresos medida a través de los ratios 10/10 y 20/20 (columnas 1-6, Tabla 4), ello con un nivel de confianza del 90 y el $95 \%$, respectivamente. Considerando el APE estimado, una comuna distante a más de $150 \mathrm{~km}$ de su respectiva capital regional (e.g., Huasco en la región de Atacama, Chonchi en la región de Los Lagos, Natales en la región de Magallanes) registraría, en promedio, un ratio 20/20 de 1,03 veces superior y un índice 10/10 de 3,02 veces mayor.

Cuarto, aquellas comunas capitales de sus respectivas provincias registrarían niveles de desigualdad menores en los valores extremos de la distribución de ingresos (columnas 1-6, Tabla 4). Este resultado es contrapuesto al reportado para el índice de Gini, lo que se explicaría porque este indicador no captura los cambios entre grupos en términos de inequidad (Abdullah et al., 2015).

En quinto lugar, la evidencia reafirma, con un nivel de significancia del $1 \%$, el efecto redistributivo que generó el alto precio del cobre en aquellas comunas con mayor proporción de trabajadores que se desempeñan en el sector minero (columnas 1-6, Tabla 4). Tales resultados complementan lo encontrado por Álvarez et al. (2018), es decir, el súper ciclo del precio del cobre no solo redujo los niveles de pobreza en aquellas comunas más expuestas al shock, sino también los niveles de desigualdad entre aquella población perteneciente a los extremos de la distribución de ingresos. Finalmente, no se encuentra evidencia de que la diferencia entre el ingreso promedio del decil/quintil más rico y el más pobre sea determinada por el porcentaje de población indígena de la comuna.

En una segunda variante de análisis, el estudio de desigualdad comunal exploró el efecto rezagado del gasto municipal en educación per cápita sobre la distribución de ingresos. Al respecto, se decidió rezagar el regresor geducper_total hasta en tres periodos, los que, dada la periodicidad de la Encuesta CASEN, tendrían una extensión de entre 2 y 6 años. Las tablas 5 y 6 dan cuenta de las estimaciones de los modelos probit de respuesta fraccional y Tobit de efectos aleatorios bajo la nueva variante de análisis descrita. 
Tabla 5. Efectos parciales promedio (APE) tras la estimación probit de respuesta fraccional y Tobit de efectos aleatorios, variante gasto en educación total rezagado

\begin{tabular}{|c|c|c|c|c|c|c|}
\hline \multirow{2}{*}{ Variable dependiente: } & \multicolumn{3}{|c|}{ Gini } & \multicolumn{3}{|c|}{ Theil } \\
\hline & (1) & (2) & (3) & (4) & (5) & (6) \\
\hline \multirow[t]{2}{*}{ geducper_totalt-1 } & 0,0000 & & & $-0,00002^{*}$ & & \\
\hline & $(0,000)$ & & & $(0,000)$ & & \\
\hline \multirow{2}{*}{ geducper_totalt-2 } & & 0,00001 & & & $-0,00001$ & \\
\hline & & $(0,000)$ & & & $(0,000)$ & \\
\hline \multirow{2}{*}{ geducper_totalt-3 } & & & $-0,00001$ & & & $-0,00001$ \\
\hline & & & $(0,000)$ & & & $(0,000)$ \\
\hline \multirow[t]{2}{*}{ Inypercap } & 0,1005 & 0,0710 & $-0,0305$ & $0,7053^{* * *}$ & $0,6159^{* * *}$ & $0,4124^{* *}$ \\
\hline & $(0,138)$ & $(0,196)$ & $(0,184)$ & $(0,154)$ & $(0,176)$ & $(0,191)$ \\
\hline \multirow[t]{2}{*}{ Inypercap2 } & 0,0022 & 0,0050 & 0,0142 & $-0,047^{* * *}$ & $-0,0396 * *$ & $-0,0225$ \\
\hline & $(0,013)$ & $(0,018)$ & $(0,017)$ & $(0,014)$ & $(0,016)$ & $(0,017)$ \\
\hline \multirow[t]{2}{*}{ etnia } & $0,0719 * *$ & $0,0566^{*}$ & 0,0645 & $0,1666^{* * *}$ & $0,1494^{* * *}$ & $0,1649 * * *$ \\
\hline & $(0,031)$ & $(0,034)$ & $(0,043)$ & $(0,030)$ & $(0,032)$ & $(0,033)$ \\
\hline \multirow[t]{2}{*}{ ruralidad } & 0,0324 & 0,0377 & 0,0166 & $0,0995 * * *$ & $0,0866 * * *$ & $0,0709 * * *$ \\
\hline & $(0,023)$ & $(0,025)$ & $(0,029)$ & $(0,022)$ & $(0,023)$ & $(0,025)$ \\
\hline \multirow[t]{2}{*}{ distancia_capitalreg } & $0,0001^{* *}$ & $0,0001 * *$ & $0,0001^{* *}$ & $0,0002^{* *}$ & $0,0003^{* * *}$ & $0,0002^{* *}$ \\
\hline & $(0,000)$ & $(0,000)$ & $(0,000)$ & $(0,000)$ & $(0,000)$ & $(0,000)$ \\
\hline \multirow[t]{2}{*}{ capitalprov } & $0,0133^{* *}$ & $0,0109^{*}$ & $0,0104^{*}$ & $-0,0031$ & $-0,0062$ & $-0,0061$ \\
\hline & $(0,006)$ & $(0,006)$ & $(0,006)$ & $(0,015)$ & $(0,015)$ & $(0,016)$ \\
\hline \multirow[t]{2}{*}{ empleomineria } & $-0,0097$ & $-0,0116$ & $-0,0106$ & $-0,3811 * * *$ & $-0,3842^{2 * *}$ & $-0,3130 * * *$ \\
\hline & $(0,057)$ & $(0,061)$ & $(0,072)$ & $(0,078)$ & $(0,082)$ & $(0,087)$ \\
\hline Observaciones & 1580 & 1264 & 948 & 1580 & 1264 & 948 \\
\hline N.o de comunas & 316 & 316 & 316 & 316 & 316 & 316 \\
\hline Test estadístico de Wald & 408,6 & 265,3 & 179,9 & 302,5 & 224,8 & 127 \\
\hline
\end{tabular}

Nota: se incluyen dummies anuales no reportadas. Errores estándar entre paréntesis. ${ }^{*} p<0,1,{ }^{* *} p<0,05,{ }^{* * *} p<0,01$. Fuente: elaboración propia.

Los resultados reportados sugieren que el efecto del gasto municipal en educación por estudiante sobre la desigualdad no sería inmediato. En particular, se confirma el efecto negativo del gasto en educación sobre el índice de Theil, el cual tardaría un periodo en materializarse (columna 4, Tabla 5). En cambio, el efecto del gasto es positivo para el indicador 10/10, el que se presentaría con un rezago de dos y tres periodos (i.e., entre 4 y 6 años en promedio), siendo este impacto cada vez mayor conforme la medición 
se aleja del periodo contemporáneo (columnas 2 y 3, Tabla 6). Por otra parte, el gasto en educación parece no afectar el coeficiente de Gini (columnas 1-3, Tabla 5) ni alterar la dispersión del ingreso entre el quintil más rico y el más pobre de la distribución de ingresos comunal (columnas 4-6, Tabla 6).

Adicionalmente, las estimaciones de la nueva variante confirman gran parte de los resultados presentados. En particular, se reafirma la existencia de la curva de Kuznets para el índice de Theil, incluso si el gasto municipal en educación se rezaga dos periodos (columnas 4 y 5, Tabla 5), mientras que esta relación se invierte para el ratio 20/20 con un nivel de significancia del $1 \%$ (columnas 4-6, Tabla 6). Además, la composición étnica de la población afecta positivamente el coeficiente de Gini y el índice de Theil si el gasto en educación se retarda hasta dos y tres periodos, respectivamente.

Tabla 6. Efectos parciales promedio (APE) tras la estimación Tobit de efectos aleatorios, variante gasto en educación total rezagado

\begin{tabular}{|c|c|c|c|c|c|c|}
\hline \multirow{2}{*}{ Variable dependiente: } & \multicolumn{3}{|c|}{$10 / 10$} & \multicolumn{3}{|c|}{$20 / 20$} \\
\hline & (1) & (2) & (3) & (4) & (5) & (6) \\
\hline \multirow[t]{2}{*}{ geducper_totalt-1 } & 0,0011 & & & 0,0002 & & \\
\hline & $(0,002)$ & & & $(0,000)$ & & \\
\hline \multirow[t]{2}{*}{ geducper_totalt-2 } & & $0,0037^{*}$ & & & $-0,00001$ & \\
\hline & & $(0,002)$ & & & $(0,000)$ & \\
\hline \multirow[t]{2}{*}{ geducper_totalt-3 } & & & $0,0059^{* *}$ & & & 0,0001 \\
\hline & & & $(0,003)$ & & & $(0,000)$ \\
\hline \multirow[t]{2}{*}{ Inypercap } & $-52,0248$ & $\mid-14,8311$ & $-52,0013$ & $-71,4797^{* * *}$ & $-44,6879^{* * *}$ & $-37,4932 * * *$ \\
\hline & $(33,171)$ & $(38,490)$ & $(43,167)$ & $(5,545)$ & $(6,098)$ & $(5,760)$ \\
\hline \multirow[t]{2}{*}{ Inypercap2 } & $6,7243^{* *}$ & 3,0947 & $6,3925 *$ & $7,0195^{* * *}$ & $4,5487^{* * *}$ & $3,9186^{* * *}$ \\
\hline & $(2,948)$ & $(3,426)$ & $(3,840)$ & $(0,487)$ & $(0,537)$ & $(0,507)$ \\
\hline \multirow[t]{2}{*}{ etnia } & $-0,1071$ & $-5,1392$ & $-7,3265$ & 0,3689 & 0,6796 & 1,4161 \\
\hline & $(4,238)$ & $(4,656)$ & $(5,305)$ & $(0,892)$ & $(0,952)$ & $(0,872)$ \\
\hline \multirow[t]{2}{*}{ ruralidad } & $10,7680^{* * *}$ & $9,9001^{* * *}$ & $10,0526^{* *}$ & $1,2808^{*}$ & $1,5403^{* *}$ & $1,4987^{* *}$ \\
\hline & $(3,138)$ & $(3,392)$ & $(3,950)$ & $(0,670)$ & $(0,707)$ & $(0,678)$ \\
\hline \multirow[t]{2}{*}{ distancia_capitalreg } & $0,0306^{* *}$ & $0,0334^{* *}$ & 0,0239 & $0,0091 * * *$ & $0,0106 * * *$ & $0,0102^{* * *}$ \\
\hline & $(0,012)$ & $(0,013)$ & $(0,015)$ & $(0,003)$ & $(0,003)$ & $(0,003)$ \\
\hline \multirow[t]{2}{*}{ capitalprov } & $-2,3166$ & $-2,2948$ & $-2,4822$ & $-0,7592^{*}$ & $-0,9326 * *$ & $-0,8218^{* *}$ \\
\hline & $(1,524)$ & $(1,690)$ & $(1,917)$ & $(0,395)$ & $(0,421)$ & $(0,396)$ \\
\hline \multirow[t]{2}{*}{ empleomineria } & $-37,6405^{* * *}$ & $-36,9274^{* * *}$ & $-26,1940 * *$ & $-9,7961^{* * *}$ & $-10,2125^{* * *}$ & $-7,4230^{* * *}$ \\
\hline & $(10,456)$ & $(11,361)$ & $(12,833)$ & $(2,333)$ & $(2,458)$ & $(2,308)$ \\
\hline Observaciones & 1130 & 904 & 678 & 1555 & 1244 & 933 \\
\hline N.o de comunas & 226 & 226 & 226 & 311 & 311 & 311 \\
\hline Test estadístico de Wald & 213,3 & 122,7 & 82,25 & 697,8 & 375,5 & 334,6 \\
\hline
\end{tabular}

Nota: se incluyen dummies anuales no reportadas. Errores estándar entre paréntesis. ${ }^{*} p<0,1,{ }^{* *} p<0,05,{ }^{* * *} p<0,01$.

Fuente: elaboración propia. 
Por otra parte, la ruralidad sigue siendo un factor determinante de la desigualdad de ingresos comunal medida por el índice de Theil o los ratios 10/10 y 20/20, incluso si el gasto en educación es rezagado en tres periodos (columnas 4-6, Tabla 5; columnas 1-6, Tabla 6). A su vez, una mayor distancia a la capital regional empeora la distribución de ingresos comunal, cualquiera que sea el indicador utilizado para medirla (columnas 1-6, Tabla 5; columnas 1, 2, 4-6, Tabla 6). Mientras que la capital provincial exhibiría, en promedio, un punto adicional en su coeficiente de Gini (columnas 1-3, Tabla 5) y una reducción de o,84 veces en su ratio 20/20 (columnas 4-6, Tabla 6).

Finalmente, los resultados ratifican el efecto positivo del súper ciclo del precio del cobre sobre la desigualdad de ingresos en Chile medida por el índice de Theil y los ratios 10/10 y 20/20.

\section{Conclusiones}

A nivel global es aceptado el hecho de que la desigualdad de ingresos desmedida constituye un impedimento para avanzar en el desarrollo de una sociedad, poniendo en riesgo las bases del crecimiento económico, las ganancias en productividad, la estabilidad política y la paz social (Alesina $\mathcal{E}$ Perotti, 1996; López 83 Miller, 2008; Abdullah et al., 2015). Al respecto, la relación negativa entre dotación de capital humano y desigualdad ha sido explotada por los gobiernos para hacer frente a esta última a través de una asignación de recursos públicos cada vez mayor a la educación en todos sus niveles. A pesar de tales esfuerzos de política pública, la evidencia es contrapuesta y no concluyente sobre los potenciales beneficios de incrementar el gasto público en educación sobre la distribución de ingresos, particularmente en economías en vías de desarrollo como las latinoamericanas (De Gregorio \&3 Lee, 2002; Sylwester, 2002; Keller, 2010; Abdullah et al., 2015). Por otra parte, el escenario que enfrentan los gobiernos locales en términos de descentralización, capital social, gobernanza e institucionalidad es gravitante en su rol como promotores del desarrollo del territorio y en el combate contra las desigualdades (Beall et al., 2000; Warner, 2001; Crook, 2003; Grindle, 2004).

Al respecto, un caso digno de análisis es el chileno, país que exhibe una estabilidad macroeconómica meritoria durante los últimos treinta años, pero una pesada carga en términos de desigualdad de ingresos (Larrañaga, 1999; López y Miller, 2008; Solimano y Torche, 2008; Álvarez et al., 2018). Con base en lo anterior, la presente investigación abordó el efecto del gasto municipal en educación per cápita sobre la desigualdad de ingresos a nivel comunal en Chile. El estudio longitudinal se enfocó en cuatro métricas tradicionales de inequidad y delineó su estrategia empírica reconociendo las características propias de tales medidas. Adicionalmente, la hipótesis central de investigación fue contrastada empleando no solo el gasto total en educación por estudiante, sino además su desagregación en tres indicadores de interés. A partir del análisis de los resultados es posible concluir lo siguiente.

Puesto que el estudio hizo la distinción entre indicadores que engloban la totalidad de la distribución de ingresos y los que se focalizan en sus valores extremos, la evidencia indica que la dirección del efecto del gasto en educación per cápita sobre la desigualdad de ingresos es sensible tanto al indicador utilizado para medirla como al componente de gasto municipal empleado en el análisis. En particular, el efecto del gasto total es negativo para el índice de Theil, pero positivo para el ratio 10/10, mientras que un mayor gasto en personal educativo incrementa el coeficiente de Gini y el ratio 10/10. Adicionalmente, el gasto en inversión parece no alterar la distribución de ingresos en las comunas chilenas. Tales resultados constituyen un hallazgo interesante, ya que la variedad de métricas y la estrategia empírica seguida no es frecuente en la literatura. 
Aun cuando los resultados confirmaron la existencia de la curva de Kuznets al considerar el índice de Theil como medida de desigualdad, esta relación se revierte en aquellos indicadores que se focalizan en los extremos de la distribución de ingreso comunal (ratios 10/10 y 20/20). Ello sugiere que la brecha de ingresos entre el decil/quintil más rico y el más pobre se exacerbe en los próximos años en Chile, lo que constituye un desafío de política pública importante para las autoridades locales y nacionales.

El estudio además reveló que aquellas comunas con una mayor concentración de población indígena enfrentarían mayores inequidades en términos de ingresos, si esta es medida a través del coeficiente de Gini o el índice de Theil. Lo anterior sería crítico en regiones extremas del norte del país y en aquellas al sur de la zona central, afectando especialmente a las etnias quechua, aymara y mapuche. Por consiguiente, la política pública propuesta por el actual gobierno del presidente Sebastián Piñera para abordar el conflicto mapuche y las disparidades sociales de la región de la Araucanía debería ampliarse a otras etnias aquejadas de igual o mayor vulnerabilidad, sobre todo si el Estado chileno pretende dar respuesta a uno de los 17 objetivos de desarrollo sostenible definidos en 2015 por el PNUD (2018).

Los resultados ratificaron la existencia de un efecto positivo de la ruralidad comunal sobre la desigualdad de ingresos, incluso cuando se evaluó el efecto rezagado del gasto municipal en educación per cápita. Por ende, el carácter rural de una comuna exacerbaría las condiciones de desigualdad existentes en un municipio con alta presencia de población indígena (e.g., Camiña, Alto Biobío, Galvarino), relevando un nuevo foco de acción para el diseño de programas sociales implementados por los gobiernos chilenos.

Se vislumbró además un efecto positivo de la distancia hacia la capital regional sobre la desigualdad entre los grupos extremos de la distribución de ingreso. Esto implica que Chile requiere no solo una mayor descentralización del poder político y económico aglutinado en la región Metropolitana, sino que requiere además resolver su concentración intrarregional. Adicionalmente, la evidencia reveló una reducción sistemática de la desigualdad en aquellas municipalidades que se vieron más expuestas al súper ciclo del precio del cobre, shock exógeno que afectó positivamente a Chile durante gran parte del periodo de estudio.

Por otra parte, dentro de las limitaciones del estudio se encuentra la baja representatividad estadística de la información capturada a través de la Encuesta CASEN en zonas aisladas del territorio chileno y la intermitencia en el conjunto de comunas a las cuales se les ha aplicado el instrumento a través del tiempo, lo que genera impactos, por ejemplo, en la construcción de un panel balanceado de larga data y de mayor cobertura poblacional; además del eventual subreporte de información que los gobiernos locales realizan al SINIM.

Finalmente, el conjunto de datos utilizado no permitió evaluar el impacto de tres políticas de educación emblemáticas implementadas recientemente en Chile: la desmunicipalización de la educación pública primaria y secundaria, la carrera docente y la introducción de la gratuidad en universidades del sistema de educación superior. Sin duda, tales tópicos configuran la agenda de investigación futura. 


\section{Referencias}

Aaron, H., \& McGuire, M. (1970). Public goods and income distribution. Econometrica, 38(6), 907-920.

Abdullah, A., Doucouliagos, H., \& Manning, E. (2015). Does education reduce income inequality? A meta regression analysis. Journal of Economics Surveys, 29(2), 301-316.

Agostini, C. A., \& Brown, P. H. (2007). Desigualdad geográfica en Chile. Revista de Análisis Económico, 22(1), 3-33.

Aigner, D. I., \& Heins, A. I. (1967). On the determinants of income equality. The American Economic Review, 57(1), 175-184.

Alesina, A., \& Perotti, R. (1996). Income distribution, political instability, and investment. European Economic Review, 40(6), 1203-1228.

Altimir, O. (1994). Distribución del ingreso e incidencia de la pobreza a lo largo del ajuste. Revista de la CEPAL, 52, 7-32.

Álvarez, R., García-Marín, A., \& Ilabaca, S. (2018). Commodity price shocks and poverty reduction in Chile. Resources Policy (in-press). doi: 10.1016/j.resourpol.2018.04.004

Amarante, V., \& Jiménez, I. P. (2016). Distribución del ingreso e imposición a las altas rentas en América Latina. Cuadernos de Economía, 35(67), 39-73.

Beall, J., Crankshaw, O., \& Parnell, S. (2000). Local government, poverty reduction and inequality in lohannesburg. Environment \& Urbanization, 12(1), 107-122.

Becker, G. S. (1971). The economics of discrimination (2.a ed.). Chicago: University of Chicago Press.

Beyer, H. (1999). Educación y desigualdad de ingresos: una nueva mirada. Serie Documentos de Trabajo, documento de trabajo n.o 297. Santiago, Chile: Centro de Estudios Públicos.

Chamberlain, G. (1980). Analysis of covariance with qualitative data. The Review of Economic Studies, 47(1), 225-238.

Champernowne, D. G. (1953). A model of income distribution. The Economic lournal, 63(250), 318-351.

Cong, R. (2000). sg144: Marginal effects of the tobit model. Stata Technical Bulletin, 56, 27-34.

Contreras, D., \& Ruiz-Tagle, J. A. (1997). Cómo medir la distribución de ingresos en Chile. ¿Son distintas nuestras regiones? ¿Son distintas nuestras familias? Estudios Públicos, 65, 59-80.

Copeland, M. A. (1947). The social and economic determinants of the distribution of income in the United States. The American Economic Review, 37(1), 56-75.

Cotte, A. \& Cotrino, J. A. (2006). Crecimiento económico y distribución del ingreso en Colombia: evidencia sobre el capital humano y el nivel de educación. Cuadernos de Administración, 19(32), 337-356.

Crook, R. C. (2003). Decentralisation and poverty reduction in Africa: The politics of local-central relations. Public Administration and Development, 23(1), 77-88.

De Gregorio, .., \& Lee, J. (2002). Education and income inequality: New evidence from cross country data. Review of Income and Wealth, 48(3), 395-416.

Galor, O., \& Zeira, I. (1993). Income distribution and macroeconomics. The Review of Economic Studies, 60, 35-52.

Grindle, M. S. (2004). Good enough governance: Poverty reduction and reform in developing countries. Governance: An International Journal of Policy, Administration, and Institutions, 17(4), 525-548.

Keller, K. R. I. (2010). How can education policy improve income distribution? An empirical analysis of education stages and measures on income inequality. The Journal of Developing Areas, 43(2), 51-77.

Kudasheva, T., Kunitsa, S., \& Mukhamediyev, B. (2015). Effects of access to education and information-communication technology on income inequality in Kazakhstan. Procedia - Social and Behavioral Sciences, 191(2), 940-947.

Kuznets, S. (1955). Economic growth and income inequality. The American Economic Review, 45(1), 1-28. 
Larrañaga, O. (1999). Distribución de ingresos y crecimiento económico en Chile. Serie Reformas Económicas n.o 35. Santiago, Chile: NU CEPAL, División de Desarrollo Económico.

Lebergott, S. (1959). The shape of the income distribution. The American Economic Review, 49(3), 328-347.

López, D. (2016). Discriminación y exclusión: tendencias en las brechas étnicas de ingresos urbanos y rurales en Chile. Serie Documentos de Trabajo n.o 200. Santiago, Chile: Rimisp, Centro Latinoamericano para el Desarrollo Rural.

López, R., \& Miller, S. J. (2008). Chile: The unbearable burden of inequality. World Development, 36(12), 26792695.

Marcel, M., \& Tokman, C. (2005). ¿Cómo se financia la educación en Chile? Estudios de Finanzas Públicas. Santiago, Chile: Dirección de Presupuestos, Ministerio de Hacienda.

Marin, A., \& Psacharopoulos, G. (1976). Schooling and income distribution. The Review of Economics and Statistics, 58(3), 332-338.

Medina, F. (1998). El ingreso y el gasto como medida del bienestar de los hogares: una evaluación estadística. En Segundo Taller Regional Medición del Ingreso en las Encuestas de Hogares (LC/R.1886) (pp. 341-371). Santiago, Chile: CEPAL.

Morley, S. A. (2000). Efectos del crecimiento y las reformas económicas sobre la distribución del ingreso en América Latina. Revista de la CEPAL, 71, 23-41.

Mostajo, R. (2000). Gasto social y distribución del ingreso: caracterización e impacto redistributivo en países seleccionados de América Latina y el Caribe. Serie Reformas Económicas n.o 69, Santiago, Chile: NU CEPAL, División de Desarrollo Económico.

Mundlak, Y. (1978). On the pooling of time series and cross section data. Econometrica, 46(1), 69-85.

Newhouse, I. P. (1971). A simple hypothesis of income distribution. The Journal of Human Resources, 6(1), 51-74.

Ocampo, J. A. (1998). Distribución del ingreso, pobreza y gasto social en América Latina. Revista de la CEPAL, 65, 7-14.

OCDE (2015). In it together: Why less inequality benefits all. París: OECD Publishing.

Papke, L., \& Wooldridge, J. (1996). Econometric methods for fractional response variables with an application to $401(\mathrm{k})$ plan participation rates. Journal of Applied Econometrics, 11, 619-632.

Papke, L., \& Wooldridge, J. (2008). Panel data methods for fractional response variables with an application to test pass rates. Journal of Econometrics, 145(1), 121-133.

Perotti, R. (1992). Income distribution, politics, and growth. The American Economic Review, 82(2), 311-316.

Pigou, A. C. (1932). The economics of welfare (4.a ed.). Londres: MacMillan \& Co.

PNUD (2017). Desiguales. Orígenes, cambios y desafíos de la brecha social en Chile. Santiago, Chile: Uqbar Editores.

PNUD (2018). Objetivos de Desarrollo Sostenible. Recuperado el 5 de junio de 2018 de http://www.undp.org/ content/undp/es/home/sustainable-development-goals.html

Ram, R. (1989). Can educational expansion reduce income inequality in less-developed countries? Economics of Education Review, 8(2), 185-195.

República de Chile, Instituto Nacional de Estadísticas (2018). Resultados Censo 2017 por país, regiones y comunas. Santiago, Chile. Recuperado el 5 de junio de 2018 de: https://resultados.censo2017.cl/

Sadka, E. (1976). Social welfare and income distribution. Econometrica, 44(6), 1239-1251.

Solimano, A., \& Torche, A. (2008). La distribución del ingreso en Chile 1987-2006: análisis y consideraciones de política. Documento de trabajo n.o 480. Santiago, Chile: Banco Central de Chile.

Strassmann, W. P. (1956). Economic growth and income distribution. The Quarterly Journal of Economics, 70(3), 425-440.

Suanes, M. (2016). Inversión extranjera directa y desigualdad de los ingresos en América Latina. Un análisis sectorial. Revista de la CEPAL, (118), 49-66. 
Sylwester, K. (2002). Can education expenditures reduce income inequality? Economics of Education Review, 21(1), 43-52.

Te Velde, D. W. (2003). Foreign direct investment and income inequality in Latin America: Experiences and policy implications. Documento de Trabajo n.o 04/03. Instituto de Investigaciones Socio Económicas (IISEC), Universidad Católica Boliviana.

Thurow, L. C. (1971). The income distribution as a pure public good. The Quarterly Journal of Economics, 85(2), 327-336.

Tinbergen, I. (1957). Welfare economics and income distribution. The American Economic Review, 47(2), 490503.

Warner, M. (2001). Building social capital: The role of local government. Journal of Socio-Economics, 30(2), 187-192.

Winegarden, C. R. (1979). Schooling and income distribution: evidence from international data. Economica, 46(181), 83-87.

Wooldridge, I. M. (2002). Econometric analysis of cross section and panel data. Cambridge: The MIT Press.

Yang, I., \& Qiu, M. (2016). The impact of education on income inequality and intergenerational mobility. China Economic Review, 37,110-125.

Zurita, G., \& Dresdner, J. (2009). Diferenciales y discriminación de salarios contra la etnia Mapuche en Chile. El Trimestre Económico, 76(304(4)), 965-989. 